\title{
Continuity and discontinuity of kirigami's high-extensibility transition: A statistical-physics viewpoint
}

\author{
Midori Isobe and Ko Okumura (1) \\ Physics Department and Soft Matter Center, Ochanomizu University, 2 Chome-1-1 Otsuka, Bunkyo City, Tokyo 112-8610, Japan
}

(Received 15 March 2019; published 3 September 2019)

\begin{abstract}
Recently, kirigami's high extensibility has been understood as a transition in the force-elongation curve. In this Rapid Communication, we consider a model, which modifies our previous model, to show a striking analogy between the present theory and the Landau theory of continuous thermodynamic transitions, if we regard a rotation angle and elongation of kirigami as the order parameter and the inverse temperature, respectively. The present study points out the importance of the distinction between the discontinuity and continuity of the highextensibility transition in an elementary kirigami structure, and shows that the mechanical response of kirigami can be understood using the tools of statistical physics, which have been proved to be useful in many fields of physics.
\end{abstract}

DOI: 10.1103/PhysRevResearch.1.022001

Origami and kirigami, traditional Japanese craft techniques based on folding and/or cutting paper, have received extensive attention in scientific fields because of their potential to impart mechanical and functional properties onto sheet materials with simple patterning $[1,2]$. The resulting applications have been frequently regarded as mechanical metamaterials [3,4] or tunable mechanical devices [5]. The basic kirigami structure, formed by patterning parallel cuts on a sheet, makes sheet materials highly stretchable, which is shown even for graphene sheets $[6,7]$. The high stretchability emerges from the transition from an in-plane to out-of-plane deformation, which is accompanied by a buckling-induced rotation of each unit of the structure [8]. While other cut patterns have been studied to explore the versatile possibilities of the application of kirigami $[9,10]$, the high stretchability of kirigami has been one of the important properties of kirigami. This property has been applied to varieties of materials such as conducting nanocomposites [11], piezoelectric materials [12], metallic glass [13], and thermally responsive materials [14], and to specific devices such as stretchable strain sensors [15] and flexible film bioprobes [16]. Some researchers have focused on other available characteristics of kirigami structures. Buckling-induced rotation has been exploited for developing solar-tracking batteries [17] and dynamic shading systems [18]. Frictional and interfacial properties have been utilized for fabricating soft actuators [19] and enhancing film adhesion [20], respectively. Although widely studied from applicationoriented perspectives, the basic physical understanding of the high extensibility of kirigami is still premature, which is the focus of the present Rapid Communication.

Published by the American Physical Society under the terms of the Creative Commons Attribution 4.0 International license. Further distribution of this work must maintain attribution to the author(s) and the published article's title, journal citation, and DOI.
As already mentioned above, the high stretchability of the basic kirigami structure [see Fig. 1(a)] has been explained by a simple model based on bending energy [8]. Experimentally, this transition manifests as a transition in the force-elongation curve. The transition could be an abrupt or a smooth transition and this difference seems to be dependent on materials used for fabricating specimens and the geometries of the cut patterns, as shown in the results reported from various groups $[12,13,15]$ (see the Discussion): We provide typical experimental results for the two opposite cases in Figs. 1(b) and 1(c). An interesting issue here is whether this transition could be discussed using terminologies of thermodynamic transitions, and if this is the case, whether the high-extensibility transition is continuous or discontinuous. In fact, in our recent work [21], we showed that our previous model proposed in Ref. [8] predicts a discontinuous transition and the prediction on the ratio between the forces just before and after the jump agrees semiquantitatively with experimental data obtained from kirigami samples made of Kent paper.

Here, we generalize our previous model and show that the kirigami's high-extensibility transition can be physically identified with the Landau theory of the second-order transition $[22,23]$, if we regard a rotation angle $\theta$ and elongation $\delta$ of each unit as the order parameter and the inverse temperature, respectively. We briefly discuss the possible mechanism of the kirigami's transition becoming a discontinuous transition.

Geometry of a unit of kirigami under tension. In this study, we consider a simplified kirigami structure, whose nondeformed geometry is specified in Fig. 1(a). An elementary unit of kirigami is defined as each of $2 N$ strips, whose width, height, and thickness are respectively given by $w, d$, and $h$ with $w \gg d$ [see Fig. 1(a)]. The $(2 n-1)$ th elementary unit (from the top) is connected at the left and right edges of volume $h d^{2}$ with the $2 n$th elementary unit $(n=1, \ldots, N)$.

In the previous study, we considered two modes of deformation when the sample in Fig. 1(a) is stretched in the vertical direction. In the in-plane deformation, the central part of volume $h w d$ of the $(2 n-1)$ th $(2 n$ th) elementary unit 
(a)

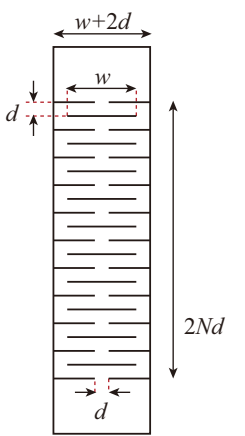

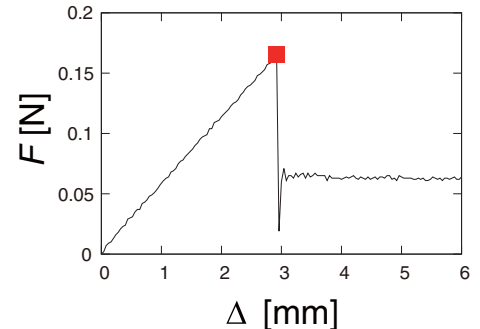

(b)
Discontinuous

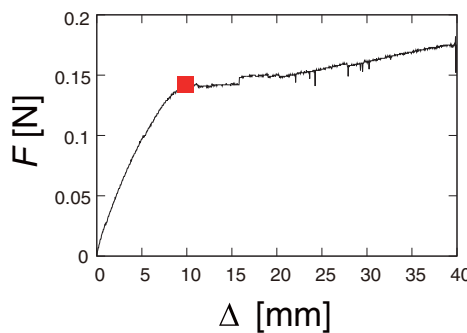

(c)

Continuous

FIG. 1. (a) Geometry of a simplified kirigami specimen. (b), (c) Two typical examples of the force $F$ as a function of the elongation $\Delta$ in the vicinity of the transition point, one exhibiting an abrupt (discontinuous) transition and the other exhibiting a continuous transition. The former is obtained from a ridged polystyrene plate of Young's modulus $E=3.1 \mathrm{GPa}(h=0.2 \mathrm{~mm}, w=40 \mathrm{~mm}, d=2 \mathrm{~mm})$, while the latter from a soft elastomer sheet of $E=7.9 \mathrm{MPa}(h=1 \mathrm{~mm}, w=30 \mathrm{~mm}, d=5 \mathrm{~mm})$.

bends "downwards" ("upwards") such that the arc of length $w$ and the center of this arc are on the original plane and the center is located below (above) the unit. In the out-of-plane deformation, the central part of volume $h w d$ of the $(2 n-1)$ th $(2 n$ th) elementary unit bends "forwards" ("backwards") such that the arc of length $w$ and the center of this arc are on the plane rotated from a horizontal plane perpendicular to the original plane by a finite (small) angle and the center is located in front of (behind) the unit.

In the present study, we allow the simultaneous existence of these two modes. (We disallow it in the previous study.) Such a general deformation is described in Fig. 2, which is explained in detail below. The in-plane and out-of-plane deformations in the previous study correspond to the special cases in which $\left(\delta_{\perp}, \theta\right)=(0,0)$ and in which $\delta_{\|}=0$, respectively.

In Fig. 2, the specimen is elongated by the amount $\Delta$ and, thus, each unit is elongated by $\delta=\Delta /(2 N)$, as shown in Fig. 2(a) and the corresponding snapshot on the left side. Here, the unit vectors in the $x, y$, and $z$ directions are given by $\hat{x}, \hat{y}$, and $\hat{z}$, respectively. In Fig. 2(a), drawn on the $x-y$ plane, the surface of the central part of volume hwd of the $(2 n-1)$ th $(2 n$ th) unit is "concave" ("convex") such that, for example, $C$ and $C^{\prime}$ are closer to you than $A$ and $A^{\prime}$ ( $A$ is closer to you than $B$ ). The side view of Fig. 2(a) is given in Fig. 2(b), which is drawn on the $y-z$ plane. A part of Fig. 2(b) is magnified in Fig. 2(c), in which the unit vectors $\hat{t}$ and $\hat{n}$ are respectively shown as the tangential and normal vectors on the surface of an above-mentioned element of kirigami, with the angle between the vector $\hat{t}$ and $\hat{y}$ being $\theta$. [Although each element is not on a plane but on a curved surface, $\hat{t}$ is identical at any point on the curved unit surface (and thus uniquely defined), and $\hat{n}$ can also be uniquely defined.] In general, for each element "rotated by the angle $\theta$ " under the given elongation $\delta$, the deformation is characterized by the

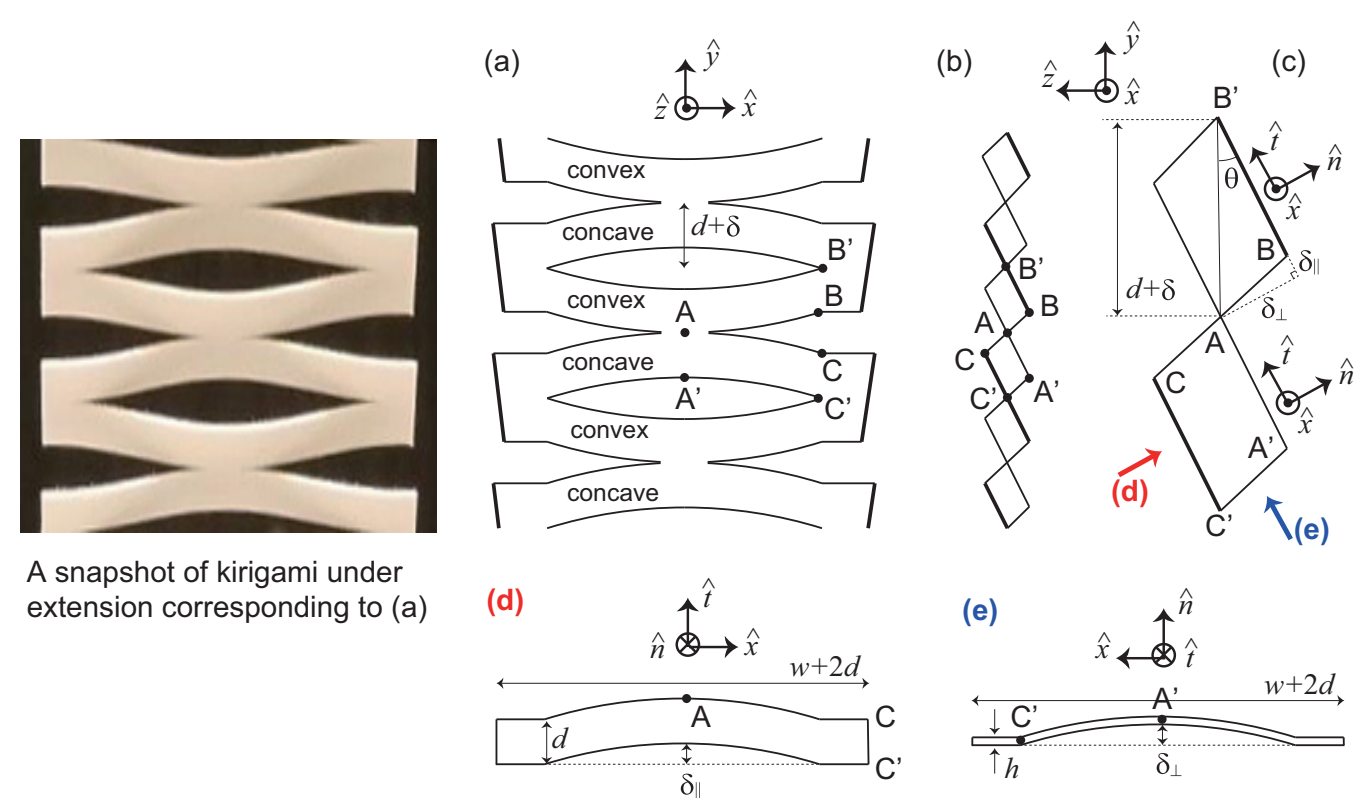

FIG. 2. Kirigami's geometry under a given elongation $\delta=\Delta /(2 N)$. In the figure, the vectors $\hat{x}, \hat{y}, \hat{t}$, and $\hat{n}$ are unit vectors, defined in the text, and used to show the plane on which the corresponding illustration is drawn. (a) Front view of an extended kirigami. (b) Side view. (c) Magnified side view. (d) The unit of kirigami containing the points $A, A^{\prime}, C$, and $C^{\prime}$ seen from the direction $\hat{n}$. (e) The same unit seen from the direction of $\hat{t}$. 
vector

$$
\vec{\delta}_{T}=\delta_{\|} \hat{t}+\delta_{\perp} \hat{n} .
$$

In Fig. 2(c), vectors $\overrightarrow{A B}, \overrightarrow{C A}$, and $\overrightarrow{C^{\prime} A^{\prime}}$ are, for example, identical to the vector $\vec{\delta}_{T}$. As clear from Fig. 2(c), $\delta_{\|}$and $\delta_{\perp}$ satisfy the following relations,

$$
\begin{gathered}
(d+\delta) \cos \theta=d+\delta_{\|}, \\
(d+\delta) \sin \theta=\delta_{\perp} .
\end{gathered}
$$

This means that as illustrated in Figs. 2(d) and 2(e), the deformation of each element can be regarded as a superposition of the bending in the $\hat{t}-x$ plane [the arc and its center characterizing the bending are on the $\hat{t}-x$ plane; see Fig. 2(d)] and the bending in the direction normal to this plane [the arc and its center are on the $\hat{n}-x$ plane; see Fig. 2(e)], which will be called "in-plane" and "out-of-plane" deformations, respectively. Note that "the plane" here does not refer to the original $x-y$ plane but the $\hat{t}-x$ plane.

Deformation energy of a unit of kirigami. From the observations we have seen in Figs. 2(d) and 2(e), according to the standard formula for the bending energy, the energy for the specified deformation per unit element is given for $w \gg d, \delta$ by

$$
\begin{gathered}
U(\delta, \theta)=U_{\|}+U_{\perp}, \\
U_{\|}=k E \frac{h d^{3}}{w^{3}} \delta_{\|}^{2}, \\
U_{\perp}=k E \frac{h^{3} d}{w^{3}} \delta_{\perp}^{2},
\end{gathered}
$$

where $E$ is Young's modulus [8,24] [see Sec. A of the Supplemental Material (SM) [25]]. In fact, the numerical coefficient $k$ depends on the boundary conditions for bending and $k=$ $(8 / 3) /\left(1-v^{2}\right)$ with $v$ Poisson's ratio [24] if we consider that the original straight line of length $w$ becomes a part of an arc assuming that $w \gg d, h$, as in the illustrations in Fig. 2. Other boundary conditions tend to increase the bending energies. (We have not considered the "net" stretching energy, which can be justified in the present case; see Sec. A of SM [25] for the details.)

The independent variables for the above energy for a given set of $w, d$, and $h$ are in fact $\delta$ and $\theta$, as seen from Eqs. (2) and (3). By renormalizing the energy and lengths by using the energy unit $k E h d^{5} / w^{3}$ and the length unit $d$, we obtain a dimensionless expression,

$$
\begin{gathered}
\tilde{U}(\delta, \theta)=\tilde{U}_{\|}+\tilde{U}_{\perp}, \\
\tilde{U}_{\|}=[(1+\tilde{\delta}) \cos \theta-1]^{2}, \\
\tilde{U}_{\perp}=\tilde{h}^{2}[(1+\tilde{\delta}) \sin \theta]^{2},
\end{gathered}
$$

with $\tilde{\delta}=\delta / d$ and $\tilde{h}=h / d$.

Transition behavior based on the energy. The energy obtained above behaves as the Landau free energy for the second-order transition, if we identify $\theta$ as the order parameter and $\delta$ as the inverse temperature. Figure 3 shows profiles of $\tilde{U}$ as a function of $\theta$ for various $\delta$ at $h / d=0.1$;

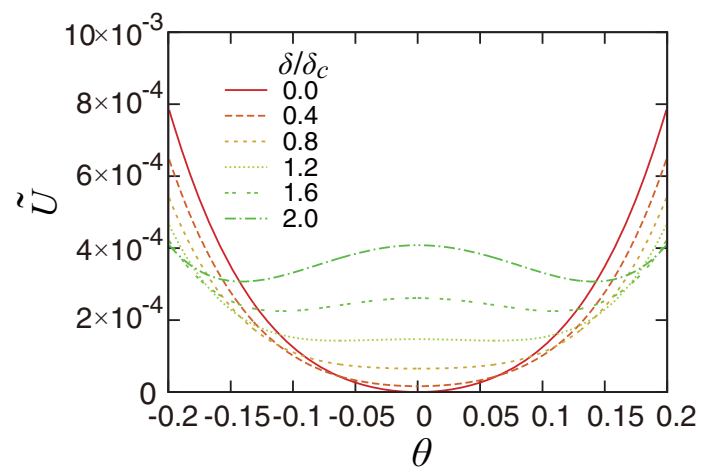

FIG. 3. Free energy $\tilde{U}$ as a function of $\theta$ for various $\delta$ at $h / d=0.1$.

at small $\delta$ the energy minimum appears at $\theta=0$ but as $\delta$ increases two minima appear symmetrically at $\theta= \pm \theta^{*}$ with a finite $\theta^{*}(>0)$, which parallels the Landau theory of critical phenomena. This feature is generic for the analytical structures as long as $h \ll d$ is satisfied, as discussed in Sec. B of $\mathrm{SM}$ [25], in which the physical origins of the emergence of Landau's scenario are elucidated. For the representation of the plots, we have introduced $\delta_{c}$ as

$$
\tilde{\delta}_{c}=\delta_{c} / d=\tilde{h}^{2} /\left(1-\tilde{h}^{2}\right) \simeq \tilde{h}^{2} .
$$

As seen in Fig. 3 [and shown precisely in Eq. (11) below], the quantity $\delta_{c}$ corresponds to "the inverse critical temperature," i.e., the value of $\delta$ at which the energy minima start to appear at nonzero values of the order parameter $\theta$. The corresponding scaling exponent is $1 / 2$, as shown in the following analytical expression derived in Sec. B of SM [25] with the assumption $\theta \ll 1$ and thus exact near the critical point for the present model,

$$
\theta^{*}= \begin{cases}0 & \text { for } \delta<\delta_{c}, \\ {\left[2\left(1-\tilde{h}^{2}\right)\left(\tilde{\delta}-\tilde{\delta}_{c}\right)\right]^{1 / 2}} & \text { for } \delta>\delta_{c} .\end{cases}
$$

Here, $\theta^{*}$ is the theoretically predicted value of the rotation angle that is obtained as the minimum of $\tilde{U}(\tilde{\delta}, \theta)$ in terms of $\theta$, by finding one of the solutions of the following equation for $\theta$,

$$
\frac{\partial \tilde{U}(\tilde{\delta}, \theta)}{\partial \theta}=0 .
$$

The order parameter in the present theory predicts a continuous transition, as seen in Eq. (11), whereas the order parameter in the previous theory $[8,21]$ predicts a discontinuous transition. As summarized in Sec. C of SM [25], the quantity $\theta^{*}$ obtained in the previous theory jumps at $\delta=2 \delta_{c}$ from $\theta=0$ to $\theta=\theta_{c}^{*}$ where $\theta_{c}^{*}$ is $\theta^{*}$ at $\delta=2 \delta_{c}$, i.e., $\theta_{c}^{*}=$ $\tan ^{-1}\left(4 \tilde{\delta}_{c}\right)^{1 / 2} \simeq 2 \tilde{h}$ [see Eq. (33) in SM [25]]. In other words, the expressions for $\theta^{*}$ in the present and previous models give the critical elongations $\delta=\delta_{c}$ and $\delta=2 \delta_{c}$, respectively, and thus the two models are similar in that the predicted critical elongations are the same at the level of scaling laws but are different because of an extra factor 2 .

The expression for $\theta^{*}$ in Eq. (11) is plotted in Fig. 4(a) under the label "Present Model (approx.)," which confirms a continuous transition. The plot labeled as "Present Model 

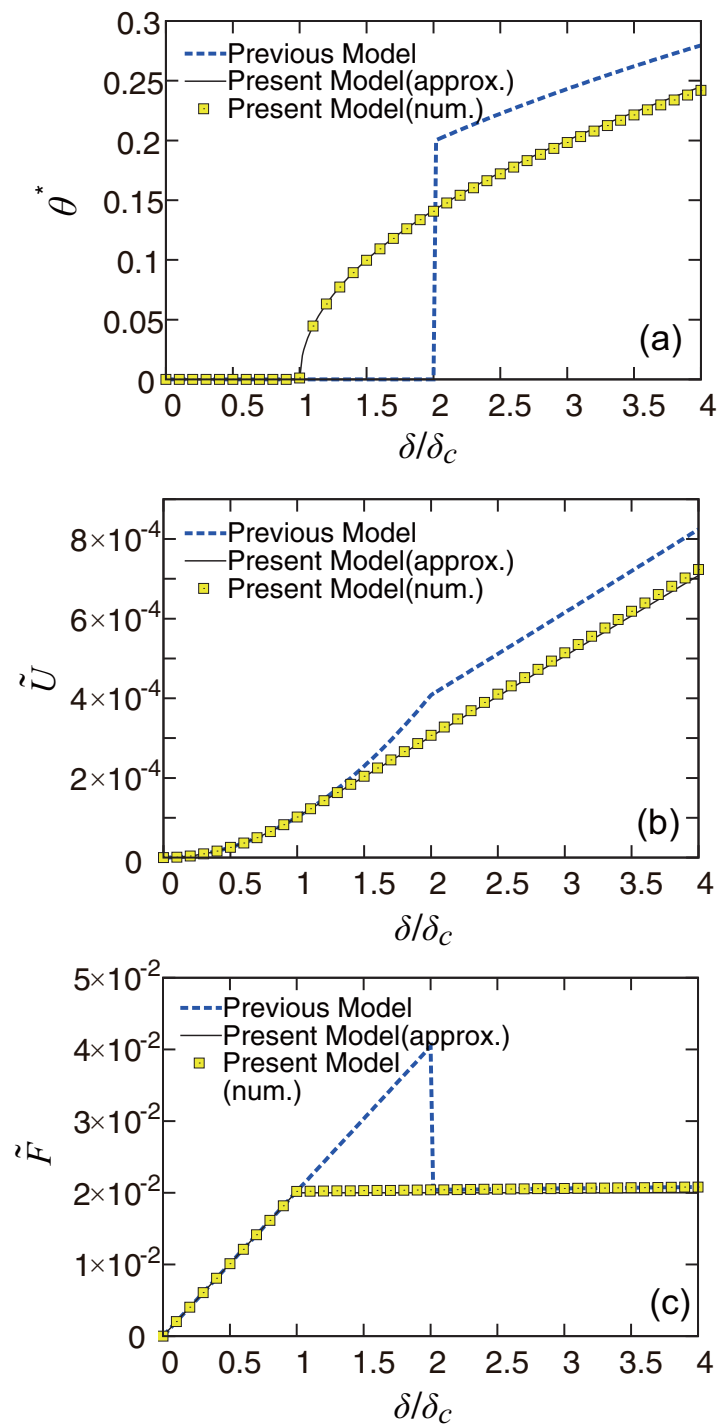

FIG. 4. (a) The value $\theta^{*}$ of "the order parameter" $\theta$ predicted by the theories as a function of "the inverse temperature" $\delta$ for $h / d=0.1$, demonstrating that the present and previous models are continuous and discontinuous, respectively. (b) Normalized free energy minimized with respect to $\theta$ as a function of $\delta$ and (c) Normalized force-elongation curve for $h / d=0.1$.

(num.)" in Fig. 4(a) is obtained numerically finding the root of the condition in Eq. (12) without using the approximation $\theta \ll 1$. The numerical and approximate plots agree well with each other, which shows that the analytical expression is a good approximation in the range of $\delta$.

The plot labeled as "Previous Model" in Fig. 4(a) is based on the analytical expression obtained in the previous model (see Sec. C of SM [25]). By comparing the three plots in Fig. 4(a), we can reconfirm the similarities and differences in the $\delta$ dependence of $\theta^{*}$ in the two models discussed above on the basis of the analytical expression.

Approximate analytical expressions in the present model for $\tilde{U}\left(\tilde{\delta}, \theta^{*}\right)$, which is $\tilde{U}(\tilde{\delta}, \theta)$ evaluated at $\theta=\theta^{*}$, and the force defined by $\tilde{F}=\left|-\partial \tilde{U}\left(\tilde{\delta}, \theta^{*}\right) / \partial \tilde{\delta}\right|$ (the force $F$ is normalized here by the unit $k E h d^{4} / w^{3}$ ) are summarized in Sec. B of SM [25]. The results are plotted in Figs. 4(b) and 4(c) under the label "Present Model (approx.)." These analytical expressions predict the following features of the present model, as confirmed by the plots: (1) $\tilde{U}\left(\tilde{\delta}, \theta^{*}\right)$ scales with $\tilde{\delta}^{2}$ and $\tilde{\delta}$ for $\delta<\delta_{c}$ and $\delta>\delta_{c}$, respectively, and the two branches are matched at $\delta=\delta_{c}$. (2) $\tilde{F}(\tilde{\delta})$ scales with $\tilde{\delta}$ and $\tilde{\delta}^{0}(=1$; independent of $\tilde{\delta})$ for $\delta<\delta_{c}$ and $\delta>\delta_{c}$, respectively, and the two branches are matched at $\delta=\delta_{c}$ (because $\tilde{\delta} \simeq \tilde{h}^{2}$ at $\delta=\delta_{c}$ for $\left.\tilde{h} \ll 1\right)$.

The plots labeled "Present Model (num.)" in Figs. 4(b) and $4(\mathrm{c})$ are, respectively, given by numerically evaluating Eq. (7) at $\theta=\theta^{*}$ [here, the value numerically obtained by solving Eq. (12)] and by differentiating the plot in Fig. 4(b) with respect to $\delta$, on the basis of the definition of $\tilde{F}$ given above. We see that the plots labeled "Present Model (approx.)" and "Present Model (num.)" in Fig. 4(b) agree with each other, as in Fig. 4(c), which justifies again our approximate analytical expressions.

Analytical expressions for $\tilde{U}$ and $\tilde{F}$ in the previous model are summarized in Sec. C of SM [25], which are plotted in Figs. 4(b) and 4(c) under the label "Previous Model." By comparing the three plots in Fig. 4(b), as well as in Fig. 4(c), we can confirm the similarities and differences summarized in the next paragraph, which can be reconfirmed through analytical expressions given in Secs. B and C of SM [25]. Note that Fig. 4(c) corresponds to the experimental results shown in Figs. 1(b) and 1(c).

The two models are similar on the following points: (1) In both models, the quadratic $\tilde{\delta}$ dependence of $\tilde{U}(\tilde{\delta})$ is switched to the linear $\tilde{\delta}$ dependence at the transition point. (2) The spring constant (slope of the force-elongation curve) before the transition is exactly the same in the two theories. However, there are important qualitative differences in the two theories. (1) In the previous theory $\tilde{U}(\tilde{\delta})$ is continuous but nonanalytic at the transition $\delta=2 \delta_{c}$ in the sense that the limit of differential coefficient from the left and that from the right are different, whereas it is analytic even at the transition $\delta=\delta_{c}$ in the present theory. (2) Accordingly, the force-elongation curve shows a discontinuous jump at the transition point in the previous theory, whereas it is continuous but nonanalytic at the transition point in the present theory; $\tilde{F}(\tilde{\delta})$ in Eq. (35) in SM [25] shows a drop from $\tilde{F}(\tilde{\delta})=4 \tilde{\delta}_{c}$ to $2 \tilde{\delta}_{c}$ at $\delta=2 \delta_{c}$, while $\tilde{F}(\tilde{\delta})$ in Eq. (32) in SM [25] shows a jumpless crossover $\left[\tilde{F}(\tilde{\delta})=2 \tilde{\delta}_{c}\right.$ at $\delta=\delta_{c} \pm \varepsilon$ with $\varepsilon$ a small number $]$.

Discussion. The previous [8] and present theories predict the same scaling laws for the spring constant in the first regime, the critical elongation, and the force at large elongation. Thus, the agreement of these predictions with our experimental data shown in Ref. [8] justifies the present theory, as well as the previous theory.

One of the important issues demonstrated in this Rapid Communication is the continuity and discontinuity in the high-extensibility transition of kirigami for the present simple slit geometry. As shown above, this distinction is captured clearly in the framework of statistical physics if we focus on the appropriate order parameter, the rotation angle, and identify the elongation with the inverse temperature.

According to several previous studies, the transition in the force-elongation curve seems to be discontinuous (e.g., Ref. [12]) or continuous (e.g., Refs. [13,15]) depending on conditions, although there has not been any systematic 
experimental investigation on the topic, except for a very recent article (but rather focusing on curvature effects for a more complex arrangement of silts) [26]. As demonstrated in Figs. 1(b) and 1(c), it has been suggested that when kirigami is made of paper or ridged plastic sheets, transitions tend to be discontinuous (corresponding to our previous model, in which the simultaneous existence of "purely in-plane and out-of-plane deformations" is disallowed), and when kirigami is made of soft gels, transitions tend to be continuous (corresponding to our present model, in which the simultaneous existence is allowed). We note here that, by developing the technique of elastic charges, the force-elongation curve has recently been discussed in Ref. [27], and the importance of stress relief is discussed in Ref. [28] focusing only on the continuous case for a rather different nonslit geometry.

To deepen our understanding of the distinction between the continuity and discontinuity, we have to explore the effects ignored in our theories, such as friction and plastic deformation, and perform experiments focusing on these aspects with systematically changing parameters, such as thickness and elastic modulus. These topics will be discussed elsewhere.

In the emerging field of the mechanics of metamaterials, the connection with critical phenomena in statistical physics has been lacking, although the scaling law (the usefulness of which is for physicists deeply rooted in the lessons learned from critical phenomena) and thermodynamic concepts have been explored in a number of recent publications [26,29] and connections to bifurcation and nonlinear physics have been stressed in the literature, probably from historical reasons [30-32]. The key words such as the critical exponent and the order parameter, which are indispensable to describe critical phenomena, have been almost absent in the literature [33], although the classic Euler buckling can be discussed in the framework of the Landau theory of critical phenomena, as explicitly demonstrated in see Sec. D of SM [25]. [A scaling relation similar to Eq. (11) is shown for kirigami actuators [34], which is again not connected to critical phenomena.] Although the present kirigami's transition is not a critical phenomenon, the remarkable analogy to the Landau theory of critical phenomena demonstrated in the present study points out to the researchers in the field the potential of the powerful tools of statistical physics, which have been proved to be useful in many fields of physics including nonequilibrium statistical physics [35].

This work was partly supported by Grant-in-Aid for Scientific Research (A) (No. 24244066) of JSPS, Japan, and by ImPACT Program of Council for Science, Technology and Innovation (Cabinet Office, Government of Japan; No. 2014-PM01-02-01). M.I. is supported by the Japan Society for the Promotion of Science Research Fellowships for Young Scientists (No. 17J04315). The authors thank Professor Edward Foley (Ochanomizu University) for valuable comments on the English.
[1] K. Miura, Method of packaging and deployment of large membranes in space, Inst. Space Astronaut. Sci. Rep. 618, 1 (1985).

[2] L. Xu, T. C. Shyu, and N. A. Kotov, Origami and kirigami nanocomposites, ACS Nano 11, 7587 (2017).

[3] S. Shan, S. H. Kang, Z. Zhao, L. Fang, and K. Bertoldi, Design of planar isotropic negative Poisson's ratio structures, Extreme Mech. Lett. 4, 96 (2015).

[4] D. M. Kochmann and K. Bertoldi, Exploiting microstructural instabilities in solids and structures: From metamaterials to structural transitions, Appl. Mech. Rev. 69, 050801 (2017).

[5] K. Bertoldi, V. Vitelli, J. Christensen, and M. van Hecke, Flexible mechanical metamaterials, Nat. Rev. Mater. 2, 17066 (2017).

[6] M. K. Blees, A. W. Barnard, P. A. Rose, S. P. Roberts, K. L. McGill, P. Y. Huang, A. R. Ruyack, J. W. Kevek, B. Kobrin, D. A. Muller, and P. L. McEuen, Graphene kirigami, Nature (London) 524, 204 (2015).

[7] Z. Qi, D. K. Campbell, and H. S. Park, Atomistic simulations of tension-induced large deformation and stretchability in graphene kirigami, Phys. Rev. B 90, 245437 (2014).

[8] M. Isobe and K. Okumura, Initial rigid response and softening transition of highly stretchable kirigami sheet materials, Sci. Rep. 6, 24758 (2016).

[9] A. Rafsanjani and K. Bertoldi, Buckling-Induced Kirigami, Phys. Rev. Lett. 118, 084301 (2017).

[10] D.-G. Hwang and M. D. Bartlett, Tunable mechanical metamaterials through hybrid kirigami structures, Sci. Rep. 8, 3378 (2018).

[11] T. C. Shyu et al., A kirigami approach to engineering elasticity in nanocomposites through patterned defects, Nat. Mater. 14, 785 (2015)
[12] N. Hu, D. Chen, D. Wang, S. Huang, I. Trase, H. M. Grover, X. Yu, J. X. J. Zhang, and Z. Chen, Stretchable Kirigami Polyvinylidene Difluoride Thin Films For Energy Harvesting: Design, Analysis, And Performance, Phys. Rev. Appl. 9, 021002 (2018).

[13] S. Chen, K. Chan, T. Yue, and F. Wu, Highly stretchable kirigami metallic glass structures with ultra-small strain energy loss, Scr. Mater. 142, 83 (2018).

[14] Y. Tang et al., Programmable kiri-kirigami metamaterials, Adv. Mater. 29, 1604262 (2017).

[15] R. Sun et al., Kirigami stretchable strain sensors with enhanced piezoelectricity induced by topological electrodes, Appl. Phys. Lett. 112, 251904 (2018).

[16] Y. Morikawa et al., Ultrastretchable kirigami bioprobes, Adv. Healthcare Mater. 7, 1701100 (2018).

[17] A. Lamoureux, K. Lee, M. Shlian, S. R. Forrest, and M. Shtein, Dynamic kirigami structures for integrated solar tracking, Nat. Commun. 6, 8092 (2015).

[18] Y. K. Yi, J. Yin, and Y. Tang, Developing an advanced daylight model for building energy tool to simulate dynamic shading device, Sol. Energy 163, 140 (2018).

[19] A. Rafsanjani, Y. Zhang, B. Liu, S. M. Rubinstein, and K. Bertoldi, Kirigami skins make a simple soft actuator crawl, Sci. Robotics 3, eaar7555 (2018).

[20] R. Zhao, S. Lin, H. Yuk, and X. Zhao, Kirigami enhances film adhesion, Soft Matter 14, 2515 (2018).

[21] M. Isobe and K. Okumura, Discontinuity in the in-plane to outof-plane transition of kirigami, J. Phys. Soc. Jpn. 88, 025001 (2019).

[22] J. Cardy, Scaling and Renormalization in Statistical Physics (Cambridge University Press, Cambridge, UK, 1996), Vol. 5. 
[23] N. Goldenfeld, Lectures on Phase Transitions and the Renormalization Group (CRC Press, Boca Raton, FL, 2018).

[24] L. Landau and E. Lifshitz, Elasticity Theory (Pergamon, Oxford, UK, 1975).

[25] See Supplemental Material at http://link.aps.org/supplemental/ 10.1103/PhysRevResearch.1.022001 for further details on the effect of stretching, analytical expressions of the present and previous theories, and Euler buckling viewed as the Landau theory of critical phenomena.

[26] A. Rafsanjani, L. Jin, B. Deng, and K. Bertoldi, Propagation of pop ups in kirigami shells, Proc. Natl. Acad. Sci. USA 116, 8200 (2019).

[27] M. Moshe, E. Esposito, S. Shankar, B. Bircan, I. Cohen, D. R. Nelson, and M. J. Bowick, Nonlinear mechanics of thin frames, Phys. Rev. E 99, 013002 (2019).

[28] M. Moshe, E. Esposito, S. Shankar, B. Bircan, I. Cohen, D. R. Nelson, and M. J. Bowick, Kirigami Mechanics as Stress Relief by Elastic Charges, Phys. Rev. Lett. 122, 048001 (2019).
[29] Y. Yang, M. A. Dias, and D. P. Holmes, Multistable kirigami for tunable architected materials, Phys. Rev. Mater. 2, 110601 (2018).

[30] A. B. Pippard, Response and Stability: An Introduction to the Physical Theory (Cambridge University Press, Cambridge, UK, 1985).

[31] B. Audoly and Y. Pomeau, Elasticity and Geometry: From Hair Curls to the Non-linear Response of Shells (Oxford University Press, Oxford, UK, 2010).

[32] Z. Bažant and L. Cedolin, Stability of Structures: Elastic, Inelastic, Fracture and Damage Theories (World Scientific, Singapore, 2010).

[33] J. Bobnar et al., Euler strut: A mechanical analogy for dynamics in the vicinity of a critical point, Eur. J. Phys. 32, 1007 (2011).

[34] M. A. Dias et al., Kirigami actuators, Soft Matter 13, 9087 (2017).

[35] R. Livi and P. Politi, Nonequilibrium Statistical Physics: A Modern Perspective (Cambridge University Press, Cambridge, UK, 2017). 